\title{
ORIGINAL ARTICLE \\ 3,5-Dicaffeoylquinic acid protects H9C2 cells against oxidative stress-induced apoptosis via activation of the PI3K/Akt signaling pathway
}

\author{
Yi-ming Bi,2, Yu-ting Wu ${ }^{1,2}$, Ling Chen ${ }^{1,2}$, Zhang-bin Tan',2, Hui-jie Fan ${ }^{1,2}$, Ling-peng Xie ${ }^{1,2}$,

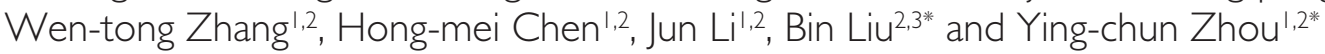 \\ ISchool of Traditional Chinese Medicine, Southern Medical University, Guangzhou, China; ${ }^{2}$ Department of Traditional \\ Chinese Medicine, Nanfang Hospital, Southern Medical University, Guangzhou, China; ${ }^{3}$ Guangzhou Institute of \\ Cardiovascular Disease, The Second Affiliated Hospital of Guangzhou Medical University, Guangzhou, China
}

\section{Abstract}

Background: Oxidative stress-induced apoptosis plays an important role in the development of heart failure. 3,5-Dicaffeoylquinic acid (3,5-diCQA), a phenolic compound, has shown protective effects against oxidative stress in many diseases.

Objective: The objective of this study was to investigate the anti-apoptosis potential of 3,5-diCQA in cardiomyocyte cells under oxidative stress and explore its underlying mechanisms.

Design: A model of tert-butyl hydroperoxide (TBHP)-induced apoptosis in a cardiomyocyte cell line (H9C2) was established. Cell viabilities on cell lines were determined by 3-(4,5-dimethylthiazol-2-yl)-2,5-diphenyl tetrazolium (MTT) assay. The apoptosis was measured by hoechst33342 and propidium iodide (PI) fluorescent staining. PI (in red) stained the regions of cell apoptosis; Hoechet33342 (in blue) stained the nuclei. The Western blot was used to determine the expressions of related proteins such as p-PI3K: phosphorylated phosphatidylinositol-3-kinase (p-PI3K), phosphorylated Serine and Threonine kinase AKT (p-AKT), p-PTEN, Bcl-2, Bax, and caspase-3. Afterward, a PI3K inhibitor, LY294002, was applied to confirm the influence of the PI3K/Akt pathway on TBHP-treated cells of 3,5-diCQA. Then, H9C2 cells were pre-incubated with 3,5-diCQA alone to determine if the expression of activated PI3K/Akt signaling was mediated by 3,5-diCQA in H9C2 cells.

Results: The results showed that TBHP resulted in an increase in cardiomyocyte apoptosis, whereas 3,5-diCQA treatment protected cells from TBHP-induced apoptosis in a dose-dependent manner. Moreover, 3,5-diCQA decreased expressions of Bax and caspase- 3 but increased the phosphorylation levels of PI3K and Akt in TBHP-treated cells, which are the key molecules mediating cell survival, whereas phosphatase and tensin homologue deleted on chromosome 10 (PTEN) phosphorylation was unchanged. Importantly, pre-incubation with a PI3K inhibitor (LY294002) partly abolished the anti-apoptosis effects of 3,5-diCQA. Further, 3,5diCQA enhanced the phosphorylation levels of PI3K and Akt in H9C2 cells directly, while LY294002 attenuated the effects of 3,5-diCQA on PI3K and Akt.

Conclusion: This study suggested that 3,5-diCQA rescued myocardium from apoptosis by increasing the activation of the PI3K/Akt signaling pathway.

Keywords: 3,5-dicaffeoylquinic acid; apoptosis; oxidative stress; PI3K/Akt pathway; cardiomyocyte

Received: 6 May 20 18; Revised: 10 September 20 18; Accepted: I4 September 20 I8; Published: I2 October 20 I8

Heart failure (HF), the end-stage of various cardiovascular diseases, is a major cause of hospitalization and mortality worldwide, with an estimated death rate at first hospital admission of about 2-17\% and more than 50\% mortality within 5 years (1). One of the critical mechanisms of HF is oxidative stress-induced apoptosis, which occurs in myocardial infarction, atherosclerosis (AS) and ischemia or reperfusion. Therefore, inhibition of cardiomyocyte apoptosis is considered to be an effective strategy in the treatment of $\operatorname{HF}(2,3)$.

Chlorogenic acids (CGAs), an important and biologically active dietary polyphenol found in fruit and plants such as coffee, cherries, and apples, show their potential antiinflammatory and anti-oxidative effects in many diseases such as cardiovascular diseases and diabetes mellitus (4-6). 3,5-Dicaffeoylquinic acid (3,5-diCQA), also called 
isochlorogenic acid $A$, a major kind of CGA, is reported to be absorbed faster and metabolized more effectively than other CGAs (7). Previous studies have demonstrated that 3,5diCQA exhibits protective effects against oxidative stress, inflammation, and gene mutations (8-10). Additionally, a number of studies indicate that 3,5-diCQA may have significant antihypertensive and anti-atherogenic effects in cardiovascular disease $(11,12)$. It has also been reported that 3,5-diCQA has protective effects on the heart (13). Moreover, treatment with 3,5-diCQA attenuated oxidative stressinduced apoptosis in vivo (14). Thus, 3,5-diCQA has become an attractive pharmacological treatment option for protecting cardiovascular cells from damage. Thus, in this study, we investigated the activity of 3,5-diCQA on cardiomyocyte apoptosis, which is one of the most important biological processes controlling $\mathrm{HF}$, and explored the further mechanisms of 3,5-diCQA on regulating apoptosis in view of signal transduction.

In the cardiovascular system, the phosphatidylinositol 3-kinase (PI3K)/Akt pathway is closely related to regulation of cardiac development, angiogenesis, and apoptosis (15). Clinical studies found that a switch toward up-regulation of Akt is associated with the potential for failing myocardium in patients (16). It also reported that Akt activation in the left ventricle of AS patients undergoing coronary artery bypass grafting was beneficial in promoting both cardiomyocyte survival and its functional recovery (17). Experimental studies found that infarct size limitation and apoptosis inhibition were associated with phosphorylation of Akt, and these effects were blocked by the PI3K inhibitors LY294002 $(18,19)$. Moreover, activation of Akt reduced hydrogen peroxide-induced cell apoptosis in Ischemia/ Reperfusion injury (20). In this study, an in vitro model of cardiomyocyte apoptosis was utilized to investigate whether the PI3K/Akt pathway was involved in the anti-apoptosis actions of 3,5-diCQA. The results of this study would shed more light on the mechanisms of 3,5-diCQA, which could potentially be used as a therapeutic agent for cardiovascular disease.

\section{Material and methods}

\section{Reagents}

3,5-DiCQA with over 98\% purity was obtained from Chengdu Must Bio-technology Co. Ltd (Sichuan, China) and dissolved in dimethyl sulfoxide (DMSO) to make a stock solution. Tert-butyl hydroperoxide (TBHP) and Hoechst 33342/propidium iodide (PI) fluorescent staining kits were purchased from Sigma (St. Louis, MO, USA). Antibodies against glyceraldehyde-3-phosphate dehydrogenase, phospho-PI3K, phospho-PTEN, Akt, phospho-Akt, caspase-3, Bax, and Bcl-2 were procured from Cell Signaling Technology (Beverly, MA, USA). LY294002 was purchased from Haoyuan Chemexpress Co. Ltd (Shanghai, China).

\section{Cell culture and treatment}

H9c2 cell line was purchased from the Cell Bank of Type Culture Collection of the Chinese Academy of Sciences (Lot No. GNR 5, Shanghai, China), and cells were cultured in Dulbecco's Modified Eagle Medium (DMEM) (Gibco, Grand Island, New York, USA) supplemented with 10\% Fetal Bovine Serum (FBS) (Gibco) and $100 \mu \mathrm{g} /$ $\mathrm{mL}$ penicillin and $100 \mu \mathrm{g} / \mathrm{mL}$ streptomycin (Gibco) at $37^{\circ} \mathrm{C}$ in a humidified atmosphere at $5 \% \mathrm{CO}_{2}$ in air. Cells for the first five passages after cell thawing were utilized in the experiment. When cells were nearly $80-90 \%$ confluent, the medium was replaced with the DMEM supplemented with $2 \% \mathrm{FBS}$ for another $12 \mathrm{~h}$ before experimental procedures. For experiments, cells were pre-incubated with different doses of 3,5-diCQA for $24 \mathrm{~h}$ and TBHP $(75 \mu \mathrm{M})$ for another $4 \mathrm{~h}$. For inhibitor experiments, cells were pre-incubated with PI3K inhibitor (LY294002, $25 \mu \mathrm{M}$ ) for $1 \mathrm{~h}$ as previously described (21) and then 3,5-diCQA and TBHP (75 $\mu \mathrm{M})$. In our study, 3,5-diCQA should be firstly dissolved in DMSO and then the resulting solution in DMEM with the final concentration of DMSO less than $0.1 \%$. As a toxic agent in our study, TBHP was diluted with DMEM as well.

\section{MTT assay}

H9C2 cells were seeded in 96-well plates at a density of $1 \times 10^{4}$ cells/well and incubated with test chemicals for the indicated time. After treatment, cell viability was assessed by MTT assay following the previous description (21).

\section{Hoechst33342/PI fluorescent staining}

H9C2 cells were seeded in 24-well plates and pre-incubated with different stimulations. Following the treatment, cells were washed twice with cold Phosphate Buffer Saline (PBS) and stained with hoechst33342 for $20 \mathrm{~min}$ under dark conditions at $37^{\circ} \mathrm{C}$ and then with $1 \mu \mathrm{g} / \mathrm{mL}$ PI for another $5 \mathrm{~min}$. The cell apoptosis index was quantified by averaging cell counts in three to four randomly selected fields per plate.

\section{Western blot assay}

Expressions of proteins after different stimulations were detected by Western blot., Proteins were harvested according to the manufacturer's instruction, and their contents were measured with a BCA protein assay kit (Beyotime, Shanghai, China). Then, proteins $(30 \mu \mathrm{g})$ were separated by $10 \%$ Sodium Dodecyl Sulphate- PolyAcrylamide Gel Electrophoresis and transferred to a Polyvinylidene difluoride membrane (Millipore, Bedford, MA, USA). The membrane was blocked in 5\% Bovine Serum Albumin for $2 \mathrm{~h}$ and then incubated with the following primary antibodies overnight at $4^{\circ} \mathrm{C}(\mathrm{p}-\mathrm{PI} 3 \mathrm{~K} 1: 1,000$, p-PTEN 1:1,000, Akt 1:2,000, p-Akt 1:1,000, caspase-3 1:2,000, Bax 1:1,000, Bcl-2 1:1,000, and GAPDH 1:3,000). Next, secondary 
antibodies conjugated to horseradish peroxidase (EARTH, San Francisco, CA, USA) were added to the membrane for $2 \mathrm{~h}$ and the results were quantified by Image $\mathrm{J}$ software (The National Institutes of Health and the Laboratory for Optical and Computational Instrumentation (LOCI, University of Wisconsin), Madison, USA).

\section{Statistical analysis}

Data were presented as mean \pm standard deviation. Statistical analysis was performed by one-way ANOVA followed by least significant difference test for comparisons between several groups. A value of $p<0.05$ was considered statistically significant.

\section{Results}

Effects of 3,5-diCQA on TBHP-induced injury in H9C2 cells

As a cytotoxic agent, TBHP reduced cell vitality in a dose-dependent manner within the range of $25-100 \mu \mathrm{M}$; $75 \mu \mathrm{M}$ TBHP decreased H9C2 viability to $54.61 \pm 4.78 \%$ $(p<0.05)$, which was used to establish an apoptotic model in subsequent experiments (Fig. 1a). Additionally, to evaluate the cytotoxicity of 3,5-diCQA, H9C2 cells were pre-incubated with 3,5-diCQA $(5,10$, and $20 \mu \mathrm{M})$ for $24 \mathrm{~h}$. The results showed that cell survival in these groups was similar to that in the control group $(p>0.05)$, indicating that 3,5-diCQA has no toxicity toward H9C2 cells (Fig. 1b). To examine the protective effect of 3,5-diCQA against TBHP-induced injury, $\mathrm{H} 9 \mathrm{C} 2$ cells were preincubated with indicated doses of 3,5-diCQA $(5,10$, and $20 \mu \mathrm{M}$ ) for $24 \mathrm{~h}$ and then cells were stimulated with $75 \mu \mathrm{M}$ TBHP for another $4 \mathrm{~h}$. Cell viability was detected using the MTT assay, cell apoptotic index was measured by Hoechst 33342/PI fluorescent staining, and the expression of apoptosis-related proteins was detected by Western blotting. MTT assay showed that exposure to TBHP reduced cell viability to $57.61 \%$ and resulted in cell shrinkage and alterations of cell shape, whereas pre-incubation with different concentrations of 3,5-diCQA (5, 10, and $20 \mu \mathrm{M})$ not only restored cell morphologies but also improved cell survival by $12.48 \%, 23.74 \%$ and $32.92 \%$, respectively ( $p<0.05$; Fig. 1c and d). Hoechst 33342/PI fluorescent staining was also performed to detect the number of apoptotic cells. The red regions (PI) represent apoptosis and the blue regions (Hoechst 33324) represent the nuclei. Consistent with the MTT assay results, most cells were stained red and showed features characteristic of apoptosis after TBHP incubation. In contrast, 3,5diCQA $(5,10$, and $20 \mu \mathrm{M})$ attenuated these changes in H9C2 cells in a dose-dependent manner $(p<0.05$; Fig. 1e a

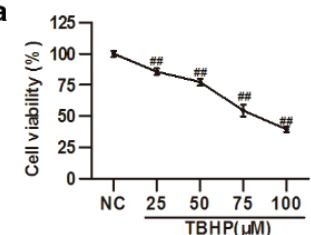

d
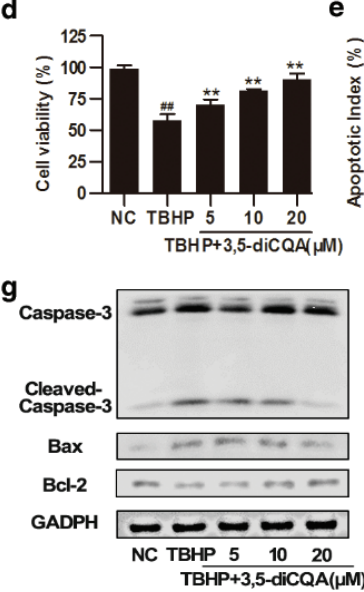
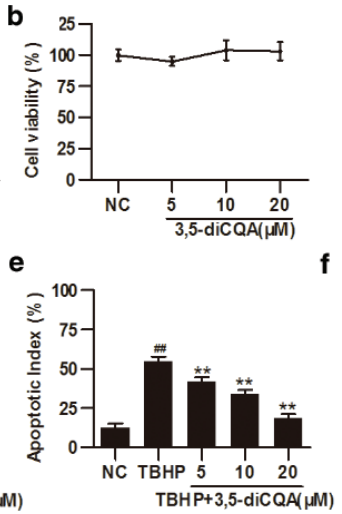

h

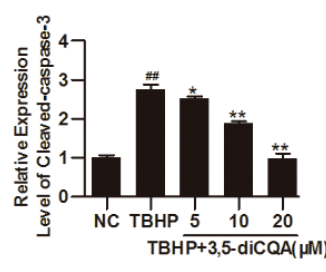

i
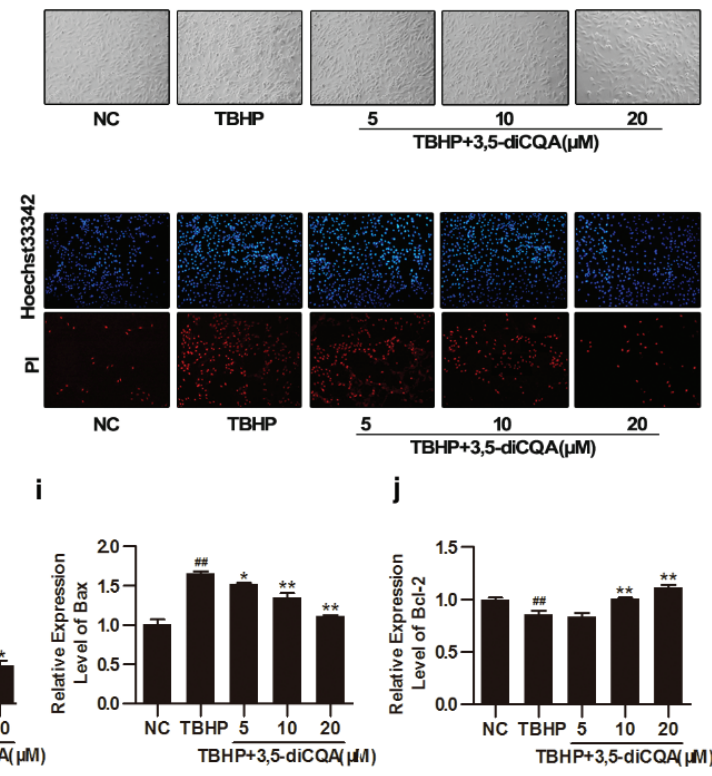

j

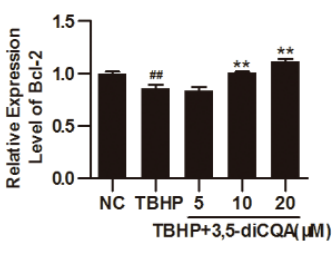

Fig. 1. Effects of 3,5-dicaffeoylquinic acid (3,5-diCQA) on tert-butyl hydroperoxide (TBHP)-induced injury in H9C2 cells. H9C2 cells were pre-incubated with the indicated doses of 3,5-diCQA $(5,10$, and $20 \mu \mathrm{M})$ for $24 \mathrm{~h}$ and then stimulated with TBHP $(75$ $\mu \mathrm{M})$ for $4 \mathrm{~h}$. Cell viability of H9C2 cells was measured by MTT assay. (a) Cytotoxicity of TBHP $(n=6)$; (b) cytotoxicity of 3,5diCQA $(n=6)$; (c) representative bright field images of H9C2 cells; (d) cell viability of 3,5-diCQA on TBHP-induced apoptosis $(n=6)$; (e and f) percentage of apoptotic cells of H9C2 determined by Hoechst 33342/ PI staining $(n=3)$; (g) Western blotting of apoptosis-related proteins including cleaved caspase-3, Bax, and Bcl-2 and their quantification (h through $\mathrm{j}$ ) ( $n=3$ ). Results were expressed as means $\pm \mathrm{SD}$. $\# p<0.05, \# \# p<0.01$ vs. normal control (NC); ${ }^{*} p<0.05,{ }^{*} p<0.01$ vs. TBHP group. 
and $\mathrm{f}$ ). To determine if the levels of apoptosis-related proteins such as caspase-3, Bax, and Bcl-2 changed, we performed Western blotting. Cleaved caspase-3, as the final effector of apoptosis, was up-regulated after TBHP incubation, while 3,5-diCQA resulted in a dose-dependent reduction of cleaved caspase- 3 expression $(p<0.05$; Fig. $1 \mathrm{~g}$ and $\mathrm{h}$ ). Meanwhile, 3,5-diCQA reduced the expression level of the pro-apoptotic protein Bax but increased the expression level of anti-apoptotic protein $\mathrm{Bcl}-2$, indicating that the ratio of $\mathrm{Bcl}-2$ to $\mathrm{Bax}$ was enhanced by 3,5diCQA compared to TBHP ( $p<0.05$; Fig. 1g through j). All these results indicated that TBHP-induced apoptosis in $\mathrm{H} 9 \mathrm{C} 2$ cells and 3,5-diCQA could inhibit such apoptotic activity of TBHP.

\section{Effects of 3,5-diCQA on PI3K/Akt signaling pathway in H9C2} cells exposed to TBHP

The PI3K/Akt signal pathway plays an important role in cardioprotection and survival (15). In order to explore the mechanisms of 3,5-diCQA in protecting H9C2 from TBHP-induced injury, expressions of key molecules in the PI3K/Akt signaling pathway were measured by Western blot. Akt is activated by PI3K in a phosphorylatedependent manner and termination of PI3K signaling is primarily achieved by the phosphatase PTEN. As Fig. 2 shows, compared with the control groups, the reductions of p-PI3K and $\mathrm{p}-\mathrm{AKT}$ by TBHP was remarkable $(p<0.05)$. However, the results showed increasing expressions of p-PI3K and p-AKT by 3,5-diCQA pre-incubation when compared with TBHP $(p<0.05)$, while 3,5-diCQA had no significant effect on the expression of p-PTEN $(p>0.05)$. These results suggest that 3,5-diCQA promotes the activation of PI3K/Akt signaling in cells exposed to TBHP.

\section{Effects of 3,5-diCQA in TBHP-induced injury of H9C2 cells} under inhibition of PI3K/Akt signaling pathway

To confirm the influence of the PI3K/Akt pathway on the cytoprotection of 3,5-diCQA, the effects of a PI3Kinhibitor, LY294002, were next examined. Cells were pre-incubated with $25 \mu \mathrm{M}$ LY294002 for $1 \mathrm{~h}$, co-incubated with $20 \mu \mathrm{M} 3$,5-diCQA for another $24 \mathrm{~h}$, and then finally incubated with $75 \mu \mathrm{M}$ TBHP. The levels of p-PI3K and p-AKT were measured by Western blotting. It was found that these proteins were induced by 3,5-diCQA supplementation in cells exposed to TBHP $(p<0.05)$, while LY294002 addition significantly suppressed the expressions of p-PI3K and p-AKT, resulting in $37.29 \%$ and $21.64 \%$ fold protein reduction, respectively. In addition, LY294002 alone suppressed the phosphorylations of both PI3K and AKT significantly compared with the normal control (NC) group ( $p<0.05$; Fig. 3a through c). Next, to further verify whether the anti-apoptosis effect of 3,5diCQA was blocked by LY294002 addition, cell viability, apoptotic index and the expressions of apoptosis-related proteins were detected. MTT results showed that the increased cell viability of 3,5-diCQA was impeded by LY294002 from $89.11 \pm 3.25 \%$ to $40.52 \pm 5.71 \%$ in TBHPtreated cells $(p<0.05$; Fig. 3d). Meanwhile, Hoechst 33342/PI fluorescent staining demonstrated that the addition of LY294002 increased the cell apoptosis index by $24.43 \%$ as compared to that with the 3,5-diCQA treatment ( $p<0.05$; Fig. 3e and $\mathrm{f}$ ). Consistently, addition of LY294002 exerted a similar effect on increasing both caspase-3 cleavage and Bax expressions, resulting in $111.9 \%$ and $85.21 \%$ fold protein increment, respectively, whereas it decreased Bcl-2 expression by $46.49 \%$ compared to 3,5-diCQA treatment ( $p<0.05$, Fig. 3g through j). Additionally, LY294002 alone also induced apoptosis of H9C2 cells concomitant with the increase of both the $\mathrm{Bax} / \mathrm{Bcl}-2$ ratio and caspase-3 cleavage compared with the $\mathrm{NC}$ group $(p<0.05)$. All the results suggested that inhibition of PI3K/Akt signaling pathway partly blocked the anti-apoptosis effect of 3,5-diCQA.

\section{Effects of 3,5-diCQA on the expression of activated PI3K/Akt signaling mediators in $\mathrm{H} 9 \mathrm{C} 2$ cells}

Next, to further study the effects of 3,5-diCQA on the expression of activated PI3K/Akt signaling, H9C2 cells were pre-incubated with 3,5-diCQA $(5,10,20 \mu \mathrm{M})$ for $24 \mathrm{~h}$ and $\mathrm{p}-\mathrm{PI} 3 \mathrm{~K}$ and $\mathrm{p}$-Akt were detected. The results of the Western blot showed that 3,5-diCQA promoted phosphorylations of PI3K and Akt dose-dependently $(p<0.05$,
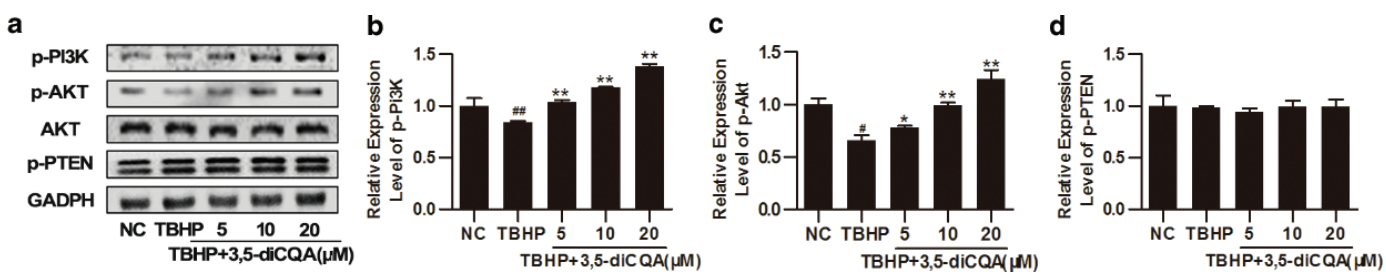

Fig. 2. Effects of 3,5-diCQA on phosphatidylinositol 3-kinase (PI3K)/Akt signaling pathway in H9C2 cells exposed to TBHP. H9C2 cells were pre-incubated with the indicated dose of 3,5-diCQA $(5,10$, and $20 \mu \mathrm{M})$ for 24 h and then stimulated with TBHP $(75 \mu \mathrm{M})$ for $4 \mathrm{~h}$. (a) Western blot was performed to demonstrate the expression of p-PI3K, p-Akt, and p-PTEN, and densities of the bands were quantified by densitometry analysis (b through $\mathrm{d})(n=3)$. Data were shown as mean $\pm \mathrm{SD}$. \#p<0.05, \#\#p<0.01 vs. normal control (NC); ${ }^{*} p<0.05, * * p<0.01$ vs. TBHP group. 
a

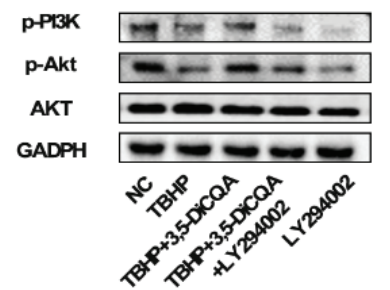

e
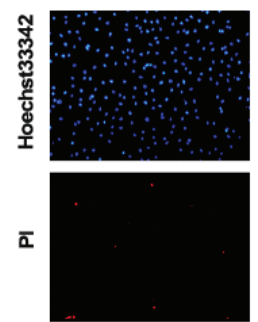

NC
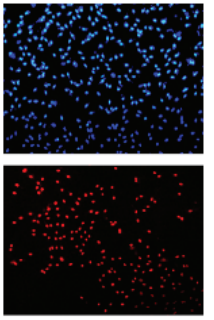

TBHP b
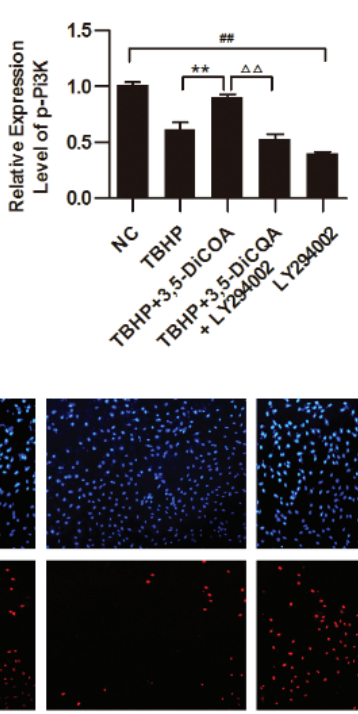

TBHP+3,5-DiCQA
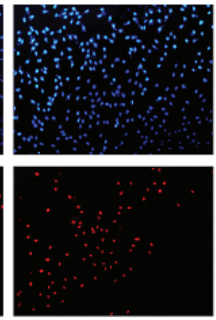

TBHP+3,5-DICQA +LY294002
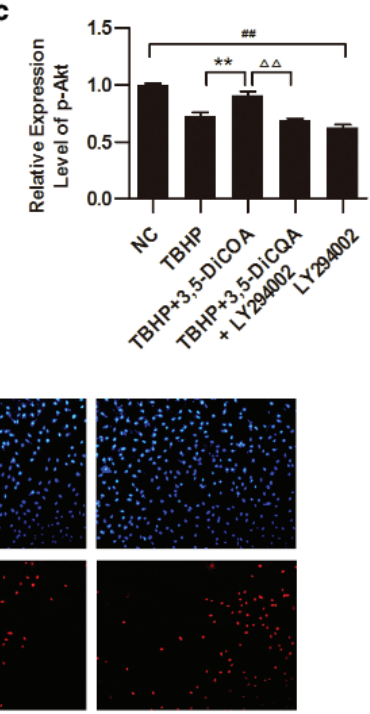

LY294002

i

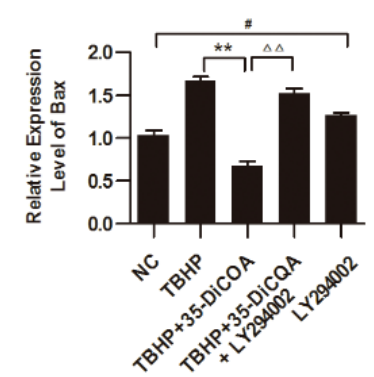

d

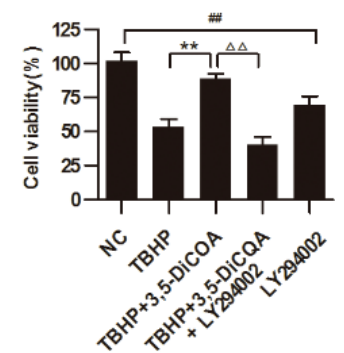

f

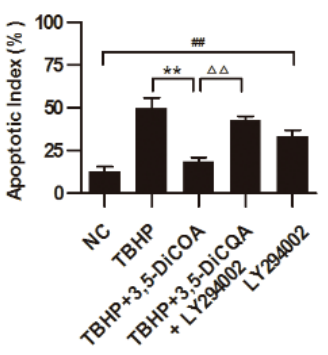

j

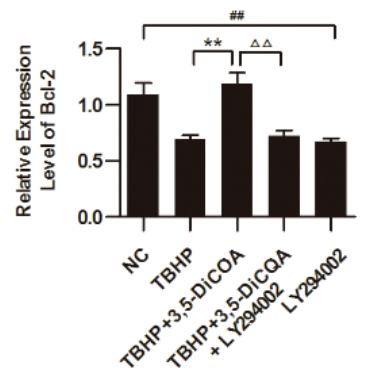

Fig. 3. Effects of 3,5-diCQA on TBHP-induced injury of H9C2 cells under inhibition of the PI3K/Akt signaling pathway. LY294002 was applied to inhibit the activation of the PI3K/Akt signaling pathway in this section. H9C2 cells were pre-incubated with $25 \mu \mathrm{M}$ LY294002 for $1 \mathrm{~h}$, and $20 \mu \mathrm{M}$ 3,5-diCQA for another $24 \mathrm{~h}$ and then $75 \mu \mathrm{M}$ TBHP. (a) Western blotting of the PI3K/ Akt signaling and the fold activation data analysis (b and c) $(n=3)$; (d) cell viability of H9C2 determined by MTT assay $(n=6)$; (e and f) Percentage of apoptotic cells of H9C2 determined by Hoechst 33342/PI staining $(n=3)$; (g) Western blotting of apoptosis-related proteins including cleaved caspase-3, Bax and Bcl-2 and the fold activation data analysis (h through j) $(n=3)$. Data were shown as mean $\pm \mathrm{SD}$. $\# p<0.05$, \#\#p $<0.01$ vs. normal control $(\mathrm{NC}) ;{ }^{*} p<0.05,{ }^{* *} p<0.01$ vs. TBHP group; ${ }^{\Delta} p<0.05$, ${ }^{\Delta} p<0.01$ vs. 3,5 -DiCQA + TBHP group.

Fig. 4a through c). LY294002 alone was also applied here, which significantly reduced the phosphorylation levels of PI3K and Akt, compared with the NC group $(p<0.05)$. LY294002 also significantly reduced the increased phosphorylation levels of PI3K and Akt of 3,5-diCQA by $77.21 \%$ and $96.69 \%$, respectively ( $p<0.05$; Fig. $4 d$ through $\mathrm{f}$ ). These results demonstrated that activation of PI3K/Akt was involved in the anti-apoptosis action of 3,5-diCQA.

\section{Discussion}

In this study, we investigated the protective effects and mechanisms of 3,5-diCQA against oxidative stressinduced $\mathrm{H} 9 \mathrm{C} 2$ apoptosis. Our study showed that the anti-apoptotic effects of 3,5-diCQA are associated with the activation of the PI3K/Akt pathway.

Cardiomyocyte apoptosis plays a critical role in the aetiology and pathogenesis of HF and oxidative stress is one of the major factors that induce cardiomyocyte apoptosis (1, 22). Inhibition of cardiomyocyte apoptosis contributes to ameliorating the failing myocardium and is the basis for therapy for $\mathrm{HF}(23,24)$. 3,5-diCQA, a common derivative of coffee, was found to protect neuronal cells in neurodegenerative diseases implicated with oxidative stress $(25,26)$. A previous study also found that 3,5-diCQA possesses a cytoprotective effect against $\mathrm{H}_{2} \mathrm{O}_{2}$-induced oxidative stress on SH-SY5Y cells (27). However, the effects of 3,5-diCQA on oxidative stress-induced apoptosis of H9C2 cells are not 


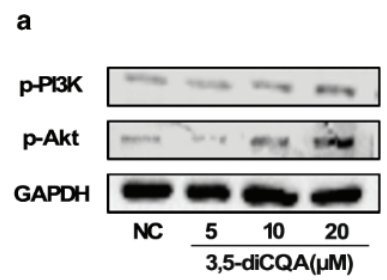

d

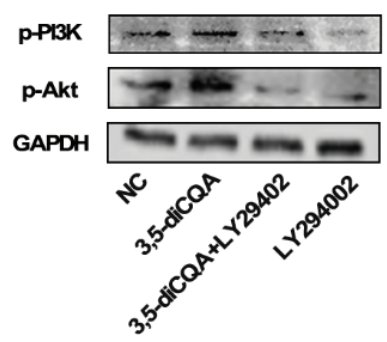

b

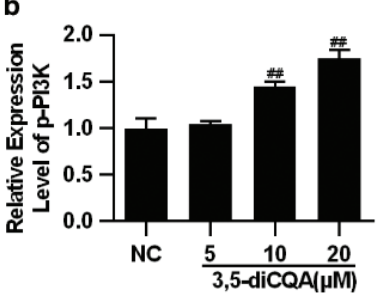

e

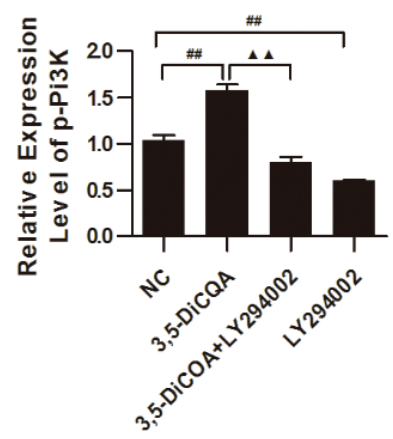

c

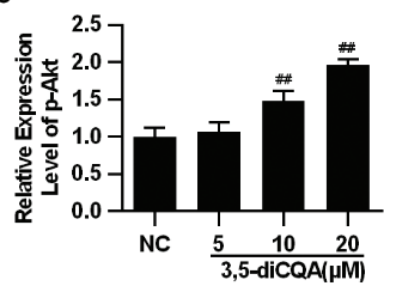

$\mathbf{f}$

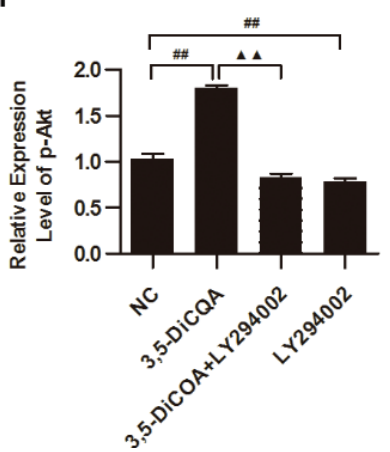

Fig. 4. Effects of 3,5-diCQA on the expression of activated PI3K/Akt signaling mediators in H9C2 cells. H9C2 cells were incubated with 3,5-diCQA $(5,10$, and $20 \mu \mathrm{M})$ for $24 \mathrm{~h}$. LY294002 was used in inhibitory experiments and H9C2 cells were preincubated with $25 \mu \mathrm{M}$ LY294002 for $1 \mathrm{~h}$, and $20 \mu \mathrm{M}$ 3,5-diCQA for another $24 \mathrm{~h}$. The indicated proteins were detected by Western blot. (a) Representative images of p-PI3K and p-Akt expression and their quantification (b and c); (d) expression of p-PI3K and p-Akt in presence of LY294002 and their quantification (e and f). The results were expressed as means \pm SD $(n=3)$. $\# p<0.05, \# \# p<0.01$ vs. normal control (NC); ${ }^{\mathbf{}} p<0.05,{ }^{\Delta}{ }^{\mathbf{\Delta}} p<0.01$ vs. 3,5-DiCQA group.

clear. Therefore, in this study, we induced the oxidative stress injury in $\mathrm{H} 9 \mathrm{C} 2$ by using TBHP, which is one of the most common oxidizing agents in experiments (28). In this study, we found that 3,5-diCQA showed a protective effect on $\mathrm{H} 9 \mathrm{C} 2$ cells with an improvement of cell viability in a dose-dependent manner, which is in agreement with previous researches $(26,27)$. These results demonstrated that 3,5-diCQA inhibits oxidative stress-induced apoptosis in $\mathrm{H} 9 \mathrm{C} 2$. The $\mathrm{H} 9 \mathrm{C} 2$ cell line of embryonic rat cardiomyocytes is derived from embryonic BD1X rat heart tissue, and HL-1 cells are a popular model for use as a cardiomyocyte cell line $(29,30)$. At the beginning of this study, we compared the backgrounds of HL-1 and $\mathrm{H} 9 \mathrm{C} 2$. We found both of these cell lines are immortalized cells with a cardiac phenotype, and both are widely used for the analysis of cardiac oxidative stress injury. However, it has been reported that H9C2 cells are more similar to primary cardiomyocytes than HL-1 cells with regard to energy metabolism patterns, such as cellular ATP levels, bioenergetics, metabolism, function, and morphology of mitochondria, and were significantly more sensitive to oxidative stress than HL-1 cells (31). Thus, we chose the $\mathrm{H} 9 \mathrm{C} 2$ cell line to establish a cell model. In addition, performing the experiment on only one cell line is a limitation of this study.

When the myocardium is subjected to oxidative stress, the metabolic and functional characteristics of the mitochondria change and the mitochondria-mediated intrinsic apoptosis pathway is initiated. The by-products of oxidative stress such as reactive oxygen species react directly with membrane lipids and proteins, causing mitochondrial dysfunction and changes of apoptotic proteins, including the Bcl-2 homology domain 3-domain interaction between Bax and $\mathrm{Bcl}-2$, release of cytochrome $\mathrm{c}$ and final activation of caspases, notably caspase- 3 , all of which induce apoptosis in cells. Furthermore, a lower ratio of Bcl-2 to Bax is associated with higher apoptosis in cells $(22,32)$. It has been reported that 3,5-diCQA attenuated caspase-3 activation induced by $\mathrm{H}_{2} \mathrm{O}_{2}$, causing an increase in survival of SH-SY5Y cells in vitro (14). Consistently, 3,5-diCQA may prevent neuronal apoptosis through the repression of apoptotic signaling molecules such as Bax in vivo (26). In this study, we found that 3,5-diCQA was indeed able to reduce apoptosis induced by TBHP in $\mathrm{H} 9 \mathrm{C} 2$ cells by improving the ratio of Bcl-2 to Bax and descending cleaved caspase-3, indicating that 3,5-diCQA inhibits apoptosis by suppression of the mitochondriamediated intrinsic apoptosis pathway.

The PI3K/Akt pathway involves the process of growth and survival in cells. It has been established that activation of the PI3K/Akt pathway by human growth factor (HGF) appears to be necessary for the anti-apoptotic effects of HGF in cardiomyocytes (32). In this study, we determined whether 3,5-diCQA performed the anti-apoptotic actions through activation of PI3K/Akt pathway. We found that the expressions of PI3K and Akt were significantly 
decreased by TBHP, whereas 3,5-diCQA dose-dependently activated the expressions of PI3K and Akt. Thus we hypothesized that the anti-apoptotic action is related to the activation of the PI3K/Akt pathway. To examine this hypothesis, a specific PI3K-inhibitor, LY294002 was applied for the next experiments. We found that LY294002 abolished the anti-apoptotic actions of 3,5-diCQA, causing low cell viability and high apoptosis, which was similar to those in TBHP. Notably, treatment cells with 3,5-diCQA alone activated PI3K and Akt directly, which were inhibited by LY294002 as well. Our results were in agreement with a previous study that 3,5-diCQA exhibits a neuroprotective effect through protection of mitochondrial activities and activation of Akt (26).

\section{Conclusion}

In summary, we found that 3,5-diCQA could inhibit apoptosis by increasing the activation of the PI3K/Akt signaling pathway in TBHP-treated $\mathrm{H} 9 \mathrm{C} 2$ cells. Our data provides new insights into the mechanisms of 3,5-diCQA in anti-apoptotic action under oxidative stress in vitro.

\section{Acknowledgements}

This work was supported by the National Natural Science Foundation of China (Grant Nos. 81673805, 81373575, and 81601779), Guangdong Natural Science Foundation (Grant No. 2014A030110210), Science and Technology Planning Project of Guangdong Province (Grant No. 2014A020221013), and Traditional Chinese Medicine Bureau of Guangdong Province (Grant No. 20161260).

\section{Conflict of interest and funding}

The authors have not received any funding or benefits from industry or elsewhere to conduct this study.

\section{References}

1. Ponikowski P, Anker SD, AlHabib KF, Cowie MR, Force TL, $\mathrm{Hu}$ S, et al. Heart failure: Preventing disease and death worldwide. ESC Heart Fail 2014;1(1):4-25.

2. Ayoub KF, Pothineni NVK, Rutland J, Ding Z, Mehta JL. Immunity, inflammation, and oxidative stress in heart failure: Emerging molecular targets. Cardiovasc Drugs Ther 2017; 31(5-6):593-608.

3. Goldenthal MJ. Mitochondrial involvement in myocyte death and heart failure. Heart Fail Rev 2016;21(2):137-55.

4. Clifford MN, Jaganath IB. Chlorogenic acids and the acyl-quinic acids: Discovery, biosynthesis, bioavailability and bioactivity. Nat Prod Rep 2017;34(12):1391-421.

5. Ghadieh HE, Smiley ZN, Kopfman MW, Najjar MG, Hake MJ, Najjar SM. Chlorogenic acid/chromium supplement rescues diet-induced insulin resistance and obesity in mice. Nutr Metabol 2015;12:19.

6. Martinez-Lopez S, Sarria B, Mateos R, Bravo-Clemente L. Moderate consumption of a soluble green/roasted coffee rich in caffeoylquinic acids reduces cardiovascular risk markers: Results from a randomized, cross-over, controlled trial in healthy and hypercholesterolemic subjects. Eur J Nutr 2018.

7. Farrell TL, Dew TP, Poquet L, Hanson P, Williamson G. Absorption and metabolism of chlorogenic acids in cultured gastric epithelial monolayers. Drug Metab Dispos 2011; 39(12):2338-46.

8. Yoshimoto M, Yahara S, Okuno S, Islam MS, Ishiguro K, Yamakawa O. Antimutagenicity of mono-, di-, and tricaffeoylquinic acid derivatives isolated from sweetpotato (Ipomoea batatas L.) leaf. Biosci Biotechnol Biochem 2002; 66(11):2336-41.

9. Iwai K, Kishimoto N, Kakino Y, Mochida K, Fujita T. In vitro antioxidative effects and tyrosinase inhibitory activities of seven hydroxycinnamoyl derivatives in green coffee beans. J Agr Food Chem 2004;52(15):4893-8.

10. Chen XM, Ma Z, Kitts DD. Effects of processing method and age of leaves on phytochemical profiles and bioactivity of coffee leaves. Food Chem 2018;249:143-53.

11. Uto-Kondo H, Ayaori M, Ogura M, Nakaya K, Ito M, Suzuki A, et al. Coffee consumption enhances high-density lipoproteinmediated cholesterol efflux in macrophages. Circ Res 2010; 106(4):779-87.

12. Dib I, Tits M, Angenot L, Wauters JN, Assaidi A, Mekhfi H, et al. Antihypertensive and vasorelaxant effects of aqueous extract of Artemisia campestris L. from Eastern Morocco. J Ethnopharmacol 2017;206:224-35.

13. Chlopcikova S, Psotova J, Miketova P, Sousek J, Lichnovsky V, Simanek V. Chemoprotective effect of plant phenolics against anthracycline-induced toxicity on rat cardiomyocytes. Part II. caffeic, chlorogenic and rosmarinic acids. Phytother Res 2004; 18(5):408-13.

14. Kim SS, Park RY, Jeon HJ, Kwon YS, Chun W. Neuroprotective effects of 3,5-dicaffeoylquinic acid on hydrogen peroxideinduced cell death in SH-SY5Y cells. Phytother Res 2005; 19(3):243-5.

15. Manning BD, Toker A. AKT/PKB signaling: Navigating the network. Cell 2017;169(3):381-405.

16. Adamopoulos S, Gouziouta A, Mantzouratou P, Laoutaris ID, Dritsas A, Cokkinos DV, et al. Thyroid hormone signalling is altered in response to physical training in patients with end-stage heart failure and mechanical assist devices: Potential physiological consequences? Interact Cardiovasc Thorac Surg 2013; 17(4):664-8.

17. Slagsvold KH, Moreira JB, Rognmo O, Hoydal M, Bye A, Wisloff $\mathrm{U}$, et al. Remote ischemic preconditioning preserves mitochondrial function and activates pro-survival protein kinase Akt in the left ventricle during cardiac surgery: A randomized trial. Int J Cardiol 2014;177(2):409-17.

18. Liu S, Ai Q, Feng K, Li Y, Liu X. The cardioprotective effect of dihydromyricetin prevents ischemia-reperfusion-induced apoptosis in vivo and in vitro via the PI3K/Akt and HIF-1alpha signaling pathways. Apoptosis 2016;21(12):1366-85.

19. Zhao X, Xiang Y, Cai C, Zhou A, Zhu N, Zeng C. Schisandrin $\mathrm{B}$ protects against myocardial ischemia/reperfusion injury via the PI3K/Akt pathway in rats. Mol Med Rep 2018;17(1): 556-61.

20. Chen XG, Lv YX, Zhao D, Zhang L, Zheng F, Yang JY, et al. Vascular endothelial growth factor-C protects heart from ischemia/reperfusion injury by inhibiting cardiomyocyte apoptosis. Mol Cell Biochem 2016;413(1-2):9-23.

21. Wang Z, Su G, Zhang Z, Dong H, Wang Y, Zhao H, et al. 25-Hydroxyl-protopanaxatriol protects against $\mathrm{H} 2 \mathrm{O} 2$-induced $\mathrm{H} 9 \mathrm{c} 2$ cardiomyocytes injury via PI3K/Akt pathway and 
apoptotic protein down-regulation. Biomed Pharmacother 2018;99:33-42.

22. Malaquin D, Tribouilloy C. [Epidemiology of heart failure]. Rev Prat 2010;60(7):911-15.

23. Gu M, Wang J, Wang Y, Xu Y, Zhang Y, Wu W, et al. MiR-147b inhibits cell viability and promotes apoptosis of rat $\mathrm{H} 9 \mathrm{c} 2 \mathrm{car}-$ diomyocytes via down-regulating KLF13 expression. Acta Biochim Biophys Sin 2018;50(3):288-97.

24. Yoshihisa A, Abe S, Kiko T, Kimishima Y, Sato Y, Watanabe S, et al. Association of serum zinc level with prognosis in patients with heart failure. J Card Fail 2018;24(6):375-83.

25. Gokcen BB, Sanlier N. Coffee consumption and disease correlations. Criti Rev Food Sci Nutr 2017:1-13.

26. Kang JY, Park SK, Guo TJ, Ha JS, Lee DS, Kim JM, et al. Reversal of trimethyltin-induced learning and memory deficits by 3,5-dicaffeoylquinic acid. Oxid Med Cell Longev 2016;2016: 6981595.

27. Deng J, Qi XL, Guan ZZ, Yan XM, Huang Y, Wang YL. Pretreatment of SH-SY5Y cells with dicaffeoylquinic acids attenuates the reduced expression of nicotinic receptors, elevated level of oxidative stress and enhanced apoptosis caused by beta-amyloid peptide. J Pharm Pharmacol 2013;65(12):1736-44.

28. Ewald CY, Hourihan JM, Blackwell TK. Oxidative stress assays (arsenite and tBHP) in Caenorhabditis elegans. Bio Protoc 2017;7(13): e2365.

29. Kimes BW, Brandt BL. Properties of a clonal muscle cell line from rat heart. Exp Cell Res 1976;98(2):367-81.

30. Claycomb WC, Lanson NA, Jr., Stallworth BS, Egeland DB, Delcarpio JB, Bahinski A, et al. HL-1 cells: A cardiac muscle cell line that contracts and retains phenotypic characteristics of the adult cardiomyocyte. Proc Natl Acad Sci U S A 1998;95(6): 2979-84.

31. Kuznetsov AV, Javadov S, Sickinger S, Frotschnig S, Grimm M. H9c2 and HL-1 cells demonstrate distinct features of energy metabolism, mitochondrial function and sensitivity to hypoxiareoxygenation. Biochim Biophys Acta 2015;1853(2):276-84.

32. Yu X, Song M, Chen J, Zhu G, Zhao G, Wang H, Hunag L. Hepatocyte growth factor protects endothelial progenitor cell from damage of low-density lipoprotein cholesterol via the PI3K/Akt signaling pathway. Mol Biol Rep 2010;37(5):2423-9.

\section{*Ying-chun Zhou}

School of Traditional Chinese Medicine

Southern Medical University

1838 Shatai Road, Guangzhou

Guangdong, 5 10515, China

Email:zhychun@I26.com

\section{*Bin Liu}

Guangzhou Institute of Cardiovascular Disease

The Second Affiliated Hospital of Guangzhou Medical University 250 Changgangdong Road, Guangzhou

Guangdong, 51 0260, China

Email:zhychun@I26.com;xmhoolv@I63.com 